\title{
Measurement and Modeling the Power Consumption of Router Interface
}

\author{
Jaewon AHN*, Hong-Shik PARK* \\ * Department of Electrical Engineering, Korea Advanced Institute of Science and Technology (KAIST), Daejeon, South Korea \\ anjwon@kaist.ac.kr, parkhs@ee.kaist.ac.kr
}

\begin{abstract}
Energy saving study without the power consumption measurement of network devices makes less effective or biased results than the study based on the empirical power consumption data. Thus, precise evaluation of network appliance power consumption is inevitable to develop more influential energy saving techniques. In this paper, we measure the power consumption of an edge router, CISCO 7609 router, as well as evaluate the effect of the packet size and the routing protocol onto the power consumption of an edge router. We investigate that with four different packet sizes according to RFC 2544 and three different routing protocols, none, OSPF, and BGP with the increment of link utilization. We analyse empirical data for numerous individual cases and find that the packet size are closely related to the power consumption of the edge router. Based on these results, we model the formal power consumption equation of the edge router.
\end{abstract}

Keywords - Power consumption, packet size, routing protocol, edge router, link utilization

\section{INTRODUCTION}

In last decade, with the tremendous growth of the Internet traffic, the power consumption of network devices has taken a large portion of the global power consumption. Due to that circumstance, the reduction of the power consumption of ICT devices has been the challenging issue on the research area. With the hard research and effort, GreenOSPF [2] as well as other outstanding research results are developed for the energy saving routing of the packet communication. These studies achieve the energy reduction by using the power consumption of a link or a device as a routing metric, for example, Yu et al. [5] uses the remaining energy of node as a routing metric for making a new energy-efficient routing protocol for green wireless mesh networks.

However, some of researches were studied based on the wrong or lough assumption of the precise power consumption of each network interface. The energy saving study without the exact power consumption measurement of network devices makes less effective or biased results than the study based on the empirical power consumption data. Thus, precise evaluation of network appliance power consumption is inevitable to develop more influential techniques for energy saving. Due to the importance of the measurement of exact power consumption, there are some studies [1][3][4] to measure the power consumption of a router or other network devices. However, the metric for the energy consumption routing is based on the link power consumption, so it is needed to study the power consumption of the router interface.

In this paper, we measure the power consumption of the router interface with the concern about the routing protocol and the packet size. Since the dynamic routing protocol is divided into the interior gateway protocol (IGP) and exterior gateway protocol (EGP), we choose open shortest path first (OSPF) and border gateway protocol (BGP) as representatives of IGP and EGP as the condition of the packet communication. Besides, the packet size is referred to RFC 2544 [7]. For the accurate configuration of the router interface, Ixia N2X, a network test application for the infrastructure test, is employed for generating the Internet traffic with the configuration against the type of the packet size and the link utilization. It is connected to a router, CISCO 7609 router [6], which can be employed in the edge of IP/MPLS provider and can support the bandwidth of the portal of WLAN as well as wireless mesh network. After setting up a test-bed, we investigate the power consumption of router interfaces according to packet sizes, routing protocols as the increment of the link utilization, which means the portion of the traffic volume against the maximum link capacity. After that, we analyse the measurement results from numerous cases and find that the packet size and the routing protocols are closely related to the power consumption of the edge router. Through the analysis, it is possible to draw a power consumption function of the router interface against the packet size, the link utilization and the routing protocols.

\section{RELATED WORKS}

There are some researches to measure network devices such as switches and routers. [3] measures the power consumption of the switch as well as analyse the empirical result. However, the difference between the max and min value of the power consumption of switch is just about $0.5 \mathrm{~W}$. [4] surveys a lot of the power consumption of routers and switches as well as analyses the tendency of the power consumption of devices according to the maximum throughput of devices. This research draws the meaningful result that the power efficiency against to the bandwidth is much higher in the large-scale devices, i.e. core routers. However, this research is studied on the power consumption of the router, not the router interface. Joseph et al. [1] presents the precise study of the power consumption of the router. They measure the power 
consumption of each line card of the router and draw an analytical model of the router power consumption. Despite these advances, they ignore the power consumption of the router interface. To the best of our knowledge, there is no prior work that concerns about the power consumption of the router interface with the routing protocol according to the packet size and link utilization.

\section{III.ENVIRONMENTS}

Environments around the router are very important for the precise measurement of the power consumption. ECR initiative [8] and ATIS [9] set up the environment to measure the router power consumption. The router should be evaluated at temperature of $25^{\circ} \mathrm{C} \pm 3^{\circ} \mathrm{C}$ and the relative humidity of $30 \%$ to $75 \%$. In addition, the router should be evaluated at a barometric pressure between 1020 and 812 mbar. In the AC power configuration, the router should be evaluated at 230 VAC $\pm 1 \%, 50$ or $60 \mathrm{~Hz} \pm 1 \%$. We put the router in a laboratory more than 4 hours to settle the potential temperature and humidity differences.

Fig. 1 shows the composition of the test-bed. The router, CISCO 7609, is composed of a routing engine, a line card, and one power supply unit. The power of the router passes the power meter. It measures the power consumption of the router periodically, so it is possible to find the power consumption of the router increase according to the configuration. For investigating the influence of the packet size and routing protocol, ten 1-gigabit Ethernet interfaces of CISCO 7609 router are connected to each port on Ixia N2X device. A port of the router performs as a gateway for an adjacent network. Whereas, a port of Ixia N2X can play a role as the network gateway which has a number of hosts as well as just a host by the configuration. For the accurate setting based on the route scale of a common router [9], the port configuration of Ixia $\mathrm{N} 2 \mathrm{X}$ differs from the kind of the routing protocol as follows:

- None: A host directly connected to the router interface

- OSPF: A network gateway which has 500 hosts

- BGP: A network gateway which has 10000 hosts

Because ten interfaces are utilized to measure the power consumption, the size of the routing table on the router, in the case of OSPF, is 5000 and the size of routing table on the router, in the case of $\mathrm{BGP}$, is 100000 . We set up the topologies for the packet communication between network gateways according to [8]. In case of none and OSPF, the fullmesh topology is settled for the connection between each ports. Fig. 2 describes the full-mesh topology, that is, entire ports on the system under unit (SUT) communicate to each ports on the traffic generator. Whereas we set up different topology, dualgroup partial mesh topology, in BGP routing protocol because of its characteristic. Fig. 3 shows the dual-group partial mesh topology, which means the ports on SUT communicate to the traffic generator divided into network side and user side.

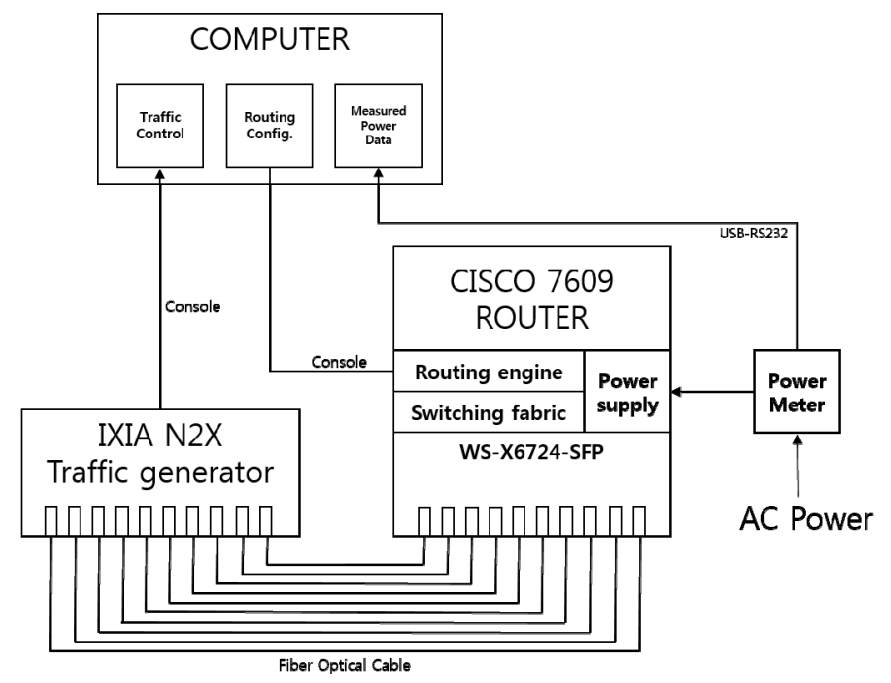

Figure 1. Composition of test-bed for measuring power consumption of the router

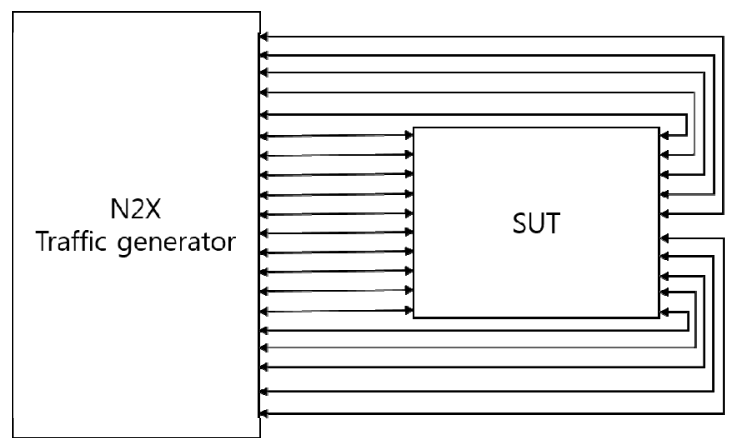

Figure 2. Full-mesh topology

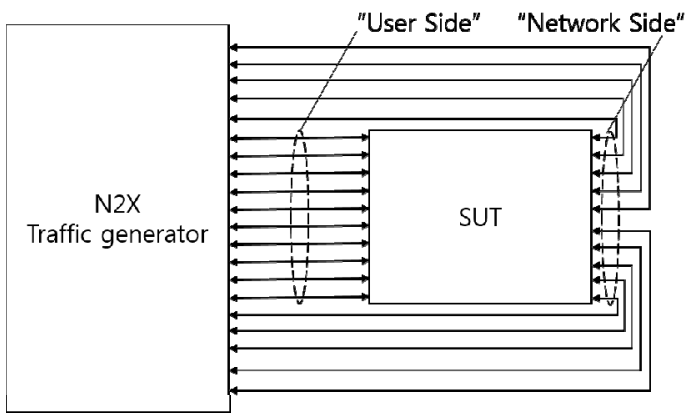

Figure 3. Dual-group partial mesh topology

\section{IV.MEASUREMENTS AND ANALYSIS}

We begin our investigation of power consumption of the router interface caused by routing protocols, packet sizes with the increment of link utilization. We measure the power consumption of router interfaces with each L2 frame size, 64, $256,512,1518$ bytes as well as each routing protocol as the increase of the link utilization. We conduct each experiment for 300 seconds and the sampling period is 5 seconds. We get the power consumption of router interface by calculating the difference between the idle state power and the measured power. The result is obtained by the average of the set of sampled power consumptions. 


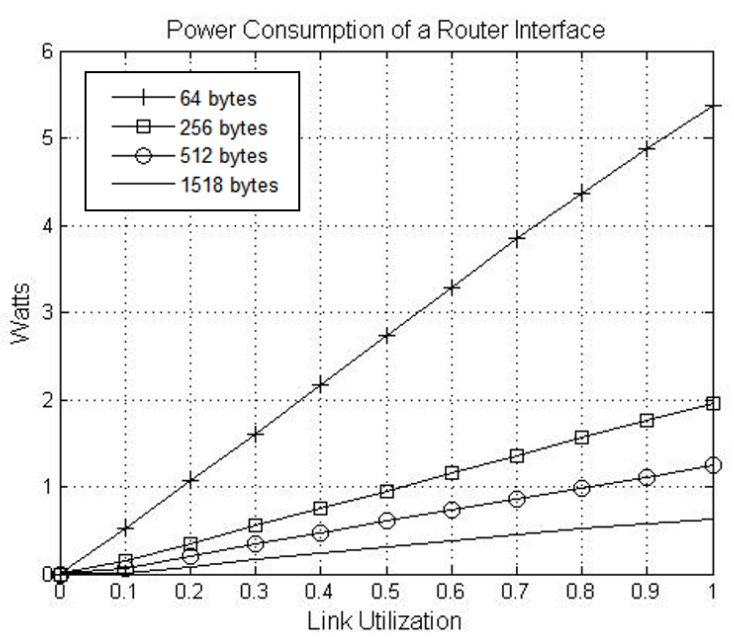

Figure 4. Power consumption of a router interface with no routing protocol

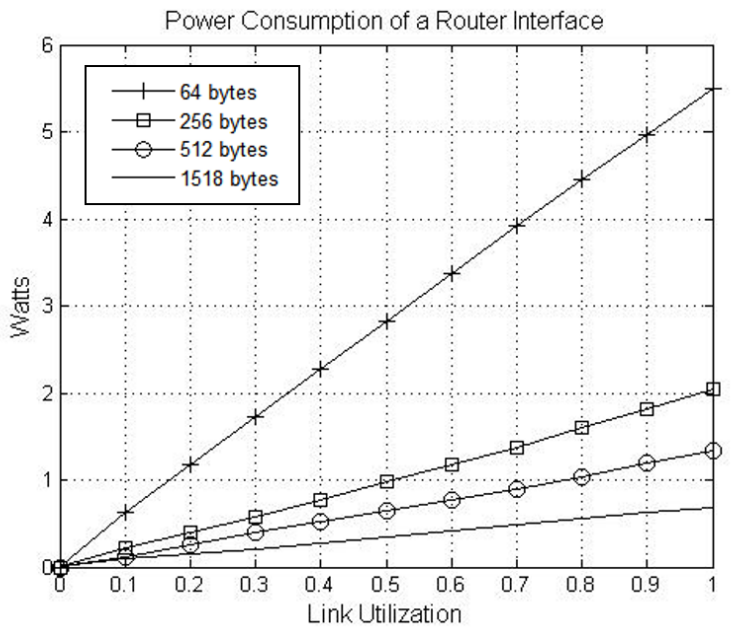

Figure 5. Power consumption of a router interface with OSPF routing protocol

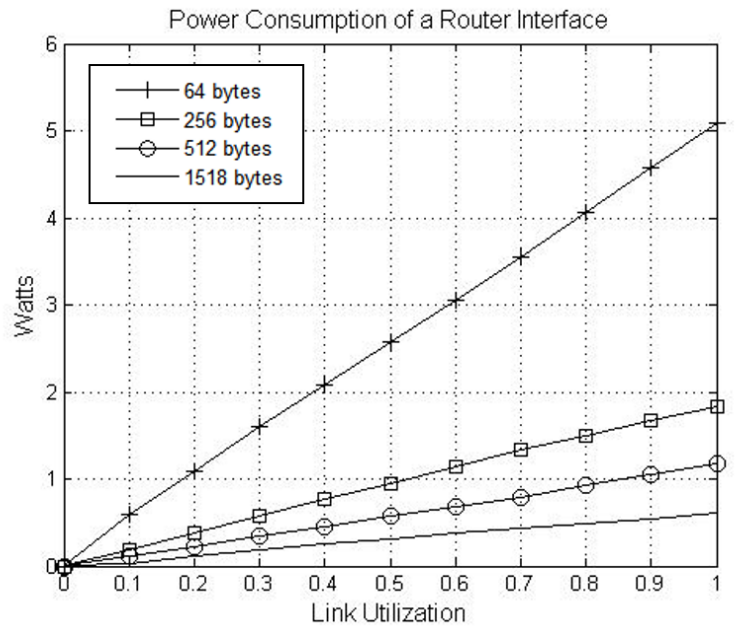

Figure 6. Power consumption of a router interface with BGP routing protocol
Fig. 4-6 shows the power consumption of the router when the generated traffic is injected to each router interface. We empirically found that the power consumption is in direct relation to the link utilization as well as the packet sizes. The power consumption increases dramatically when the traffic with 64 bytes L2 frame size. In the other cases, it also increases considerably. The power consumption of the router interface increases more than 5 watts and this value can't be ignored because a router which has $n$ interfaces consumes more than $5 n$ watts caused by just router interfaces even though the power consumption of the router is much larger than that of the router interface. It is because the router utilizes electricity for processing packets pass through the router. The more frames pass through the router, the more power is used in the router.

We also found that the power consumption of the router is slightly different from each routing protocol. It is because the increase of the power consumption caused by the routing protocol is occurred by the exchange of each routing table, however, it is temporally generated. So the increment caused by the exchange of routing table doesn't appear that much in the result, that is, the average of the sampled power consumption.

\section{MODELLING}

In the previous chapter, we found that the power consumption is in direct proportion to the link utilization as well as the packet sizes. So, it can be defined as following function, $P_{\text {interface }}(\rho, s, c)$, where $\rho$ means the link utilization, $s$ is the packet size, and $c$ means the computing coefficient which concerns the power consumption overhead by routing protocols. Though there is an increase of the power consumption when routing protocols are exchanged between routers, it appears temporally. Thus we can ignore the parameter $c$, then the power consumption function will be like $P_{\text {interface }}(\rho, s)$. In addition, we already found that the router interface consumes the power for processing incoming packets, and the power consumption of an interface can be separated to the power consumption for the header processing and the packet transferring. So it is possible to draw the power consumption function like following,

$$
\begin{aligned}
P_{\text {interface }}(\rho, s) & =P(\text { header processing })+P(\text { packet transferring }) \\
& =E_{H P} \times(\text { packets per second })+E_{P T} \times(\text { bits per second })
\end{aligned}
$$

, where $E_{H P}$ (Joule) is the packet header processing energy and $E_{P T}$ (Joule/bit) is the per bit transfer energy. Both of them depend on the property of each router interface, that is, the constant value which can be obtained by the power consumption measurement.

The power consumption of a router interface is shown according to the following equation: 


$$
\begin{aligned}
P_{\text {interface }} & =\left(E_{H P} \times \frac{\rho \times R}{s}\right)+\left(E_{P T} \times \rho \times R\right) \\
& =\rho \times R \times\left(\frac{E_{H P}}{s}+E_{P T}\right)
\end{aligned}
$$

,where $R$ is the maximum link utilization of the router interface. Because the $\mathrm{R}$ is also the constant value, the power consumption can be obtained by the packet size and the link utilization. If the packet size also be fixed to a constant value, the power consumption function of a router interface can be a linear function same as functions in the Fig. 4-6.

Besides, we can obtain the power consumption of the router interface when the traffic with multiple packet sizes passes the router by following equation,

$$
P_{\text {interface }}=\sum_{i}\left(\rho_{i} \times R \times\left(\frac{E_{H P}}{s_{i}}+E_{P T}\right)\right)
$$

,where $i$ is the number of packet sizes, $s_{i}$ is the $i$-th packet size, $\rho_{i}$ means the partial link utilization of the $i$-th packet sizes and $\sum \rho_{i}$ means $\rho$.

With the power consumption equation of a router [1], we can obtain the exact power consumption of the router with the customized configuration. For example, the router only use four router interfaces, we can get the power consumption of the router by summarizing the power consumption of its chassis, linecards, and the four individual utilized interfaces.

\section{CONCLUSIONS}

In this paper, we have measured the accurate power consumption of a router interface and drawn an analytical power consumption model of a router interface. First, we measure the power consumption of the router interface with configurations against the packet size and routing protocols. According to the result of this measurement, it is found that the power consumption of the router interface isn't an ignored value, but the important value to obtain the precise power consumption of the router. Besides, the power consumption function of the router interface can be composed of link utilization and the packet size. With the modelling, we can find that the power consumption of the router interface is in the direct proportion to the link utilization as well as in the reverse proportion to the packet size. Since the power consumption of the router interface can be a routing metric, i.e. the power consumption of a link, for achieving the energy reduction on the packet communication, the power consumption function composed of link utilization and the packet size is useful to calculate the routing metric of energy saving routing protocols. Our further work will draw the power consumption function of the router interface more precisely by measuring the power consumption of more routers.

\section{ACKNOWLEDGMENT}

This research was funded by the MSIP (Ministry of Science, ICT \& Future Planning), Korea in the ICT R\&D Program 2013.

\section{REFERENCES}

[1] Chabarek, J., et al., "Power Awareness in Network Design and Routing," INFOCOM IEEE Conference on Computer Communications, 2008 .

[2] Cianfrani, A., et al., "An Energy Saving Routing Algorithm for a Green OSPF Protocol," INFOCOM IEEE Conference on Computer Communications Workshops, 2010.

[3] Hlavacs, H., et al., "Energy Consumption of Residential and Professional Switches," Computational Science and Engineering, 2009. CSE '09. International Conference on. 2009

[4] Mellah, H. and B. Sanso. "Routers vs switches, how much more power do they really consume? A datasheet analysis," World of Wireless, Mobile and Multimedia Networks (WoWMoM), 2011 IEEE International Symposium on a.

[5] Yu, Y., et al., "A New Energy-Efficient On-Demand Routing Protocol for Green Wireless Mesh Networks,"in Proceedings of the 2012 2nd International Conference on Computer and Information Application (ICCIA 2012)

[6] CISCO 7609 router. [Online]. Available: http://www.cisco.com/

[7] RFC 2544. [Online]. Available: http:// www.ietf.org/rfc/rfc2544.txt/

[8] Network and Telecom Equipment-Energy and Performance Assessment, Draft 3.0.1, ECR Initiative, Dec. 14, 2010

[9] Energy Efficiency for Telecommunication Equipment: Methodology for Measurement and Reporting, ATIS-0600015.2009. 